\title{
Treatment of Elbow Joint Injuries
}

\author{
By L. MACE DAVID, M.B., F.R.C.S. (Ed.)
}

Clinical Assistant, Department of Surgery, Transvaal Memorial Hospital for Children.

More than 50 years ago Hugh Owen Thomas taught his students the dangers of stretching and forcibly manipulating an elbow joint which is. stiff as the result of trauma. Such treatment is still being pursued and is having the opposite effect of that for which it was intended.

The elbow joint is very susceptible to injury. Impaired function, so obvious in most cases, may be caused not only by a lesion within the joint (intraarticular) but by some condition either of the soft tissues in the vicinity of the joint or within the muscles which activate it (extra-articular). It can also be caused by minor strains if they are repeated frequently.

The commonest cause of stiffness of the joint is adhesion formation, either intra- or extra-articular. An adhesion is a pathological band resulting from exudate brought about by trauma or inflammation $\left(^{1}\right)$. Thus, after the injury, joint stiffness occurs because of adhesions of the capsular plication which is the result of organisation of the exudate in the periarterial tissues. 


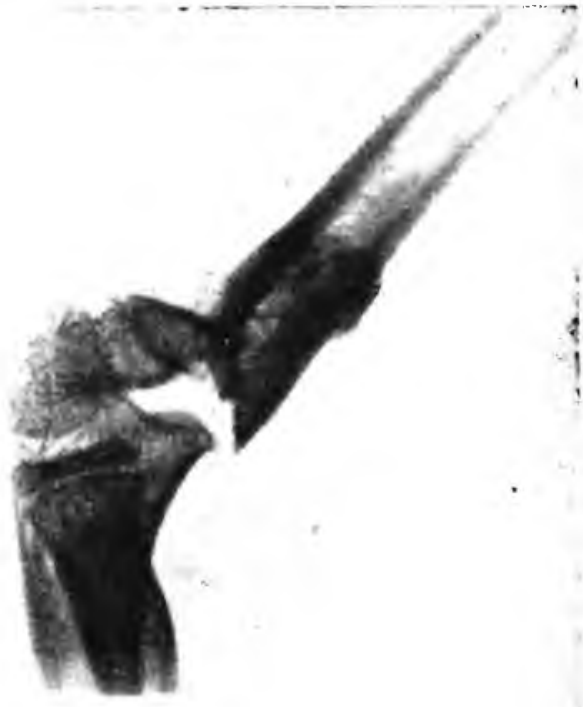

Fig. 1.-Lateral View.-Before Manipulation. Showing posterior displacement plus angulation of clistial fragment.

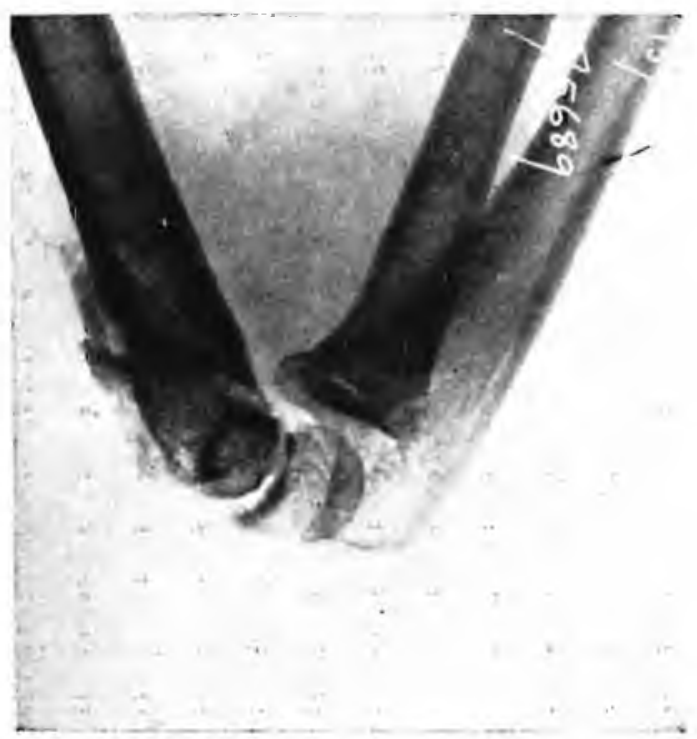

Fig. 2.-Lateral View.-After Manipulation.

Fragments now in good alignment.

Watson-Jones points out that there are several important factors which contribute to the continued or recurrent exudate.

I. Functional inactivity and disuse:-Circulatory and lymphatic stasis and waterlogging of the tissues with sero-fibrinous Auid.

2. Joint injury:-Traumatic sero-fibrinous exudation from the torn capsule or from a neighbouring fracture-a source of recurrent exudation if the injured joint is frequently or forcibly moved in the early stages.

3. Recurrent oedema :-Reactionary traumatic oedema especially in the lower limbs in the later stages.

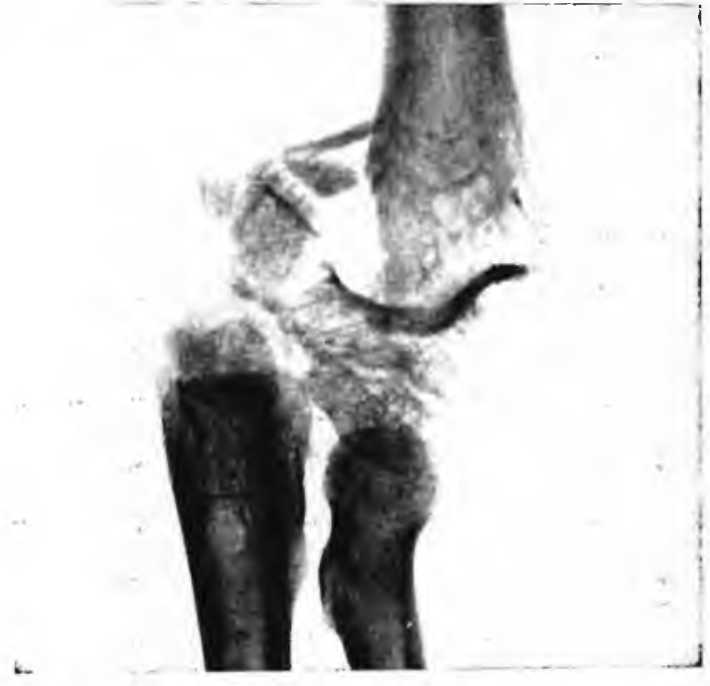

Fig. 1a.-A.P. View.-Before Manipulation.

Showing medial displaccment with inter condrlor fracture.

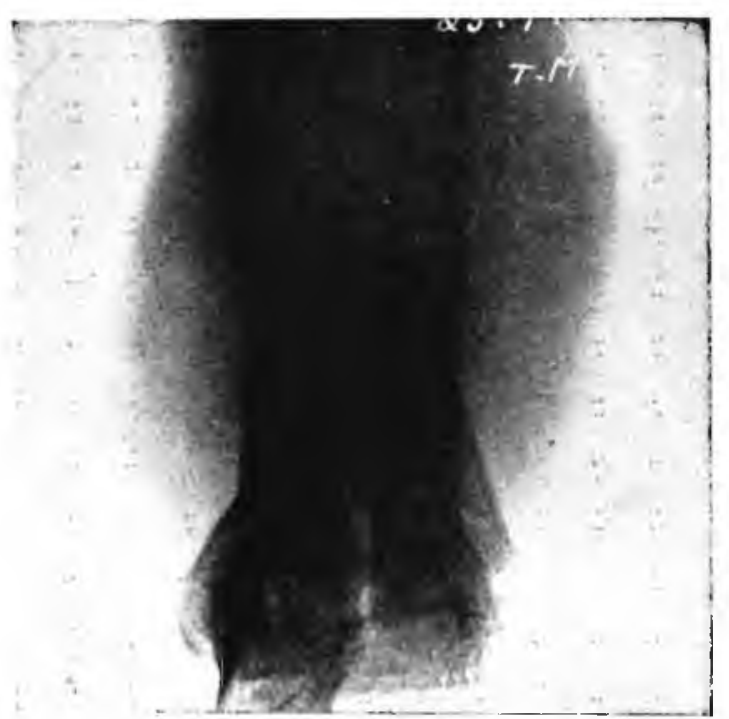

Fig. 2a.-A.P. View.-After Manipulation.

Fragnents in good aligmment.

4. Infection near the joint:-Infammatory serofibrinous exudation spreading from a neighbouring focus of infection.

5. Foreign bodies, especially skeletal traction pins close to joints:-Reactionary and low grade infammatory sero-fibrinous exudation spreading from the pin track.

6. Repeated passive stretching and forcible manipulation of it stiffened foint:-Tramatic serofibrinous exudation from the stretched and torn adhesions (').

One can discurd causes 3.4 and 5 , and concentrate on numbers 1,2 and 6 for the purposes of this article. 
Functional inactivity and disuse.-Immobilisation in itself is not a major factor in the development of aclhesions, when uncomplicated by other factors. Any adliesions so formed are due to venous and lymphatic stasis, and once the patient resumes active use of the part, recovery of normal function occurs. But if the injured joint is treated by early passive movements. there is repeated exudation and it is this which causes dense adhesions.

Joint injury.-A simple joint injury such as dislocation or uncomplicated fracture does not cause serious or lasting disability. The practice of treating dislocations and fractures by immediate movements, repeated daily, defeats its own object. Instead of preventing stiffness, it aggravates it. The correct approach is completely to immobilise the joint until all the tissues have healed, and then to start exercises by making the patient carry out active movements only. It must not be forgotten that the proximal and distal joints should be actively exercised while the injured joint is immobilised.

Repeated passive stretching and forcible manipulation of a stiffened joint.-Massage and movements, so) commonly prescribed by the practising medical attendant as the correct treatment for a stiff joint are, in fact, onc of the commonest causes of a stiff joint. When an adhesion is formed and is stretched or torn by passive stretching or manipulation, reactionar, exudation follows. producing fresh adhesions.

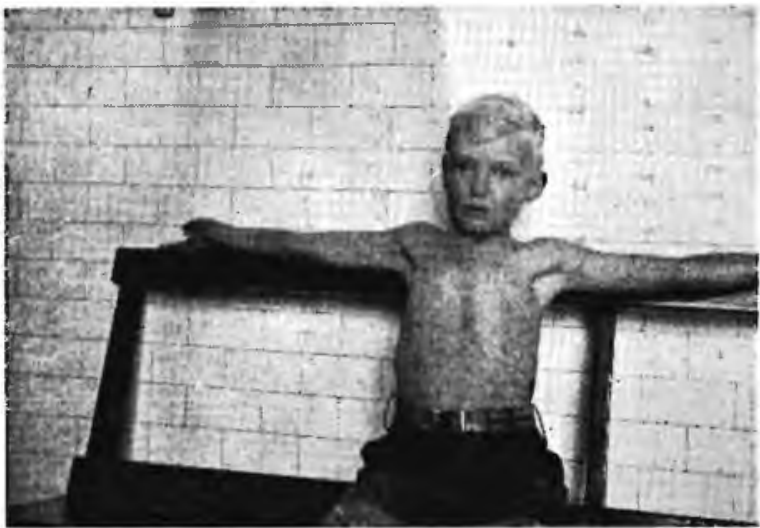

Fig. 3.-3 months after injury-Full extension recovered.

Ii this treatment is continued, it breaks and stretches some adhesions, but fresh adhesions will still be formed, retarding and delaving recovery of movement.

Enthusiastic physiotherapists seem unable to resist the temptation to force a stiff elbow joint. WatsonJoncs emplasises not only the serious disability that follows sucli action, but also denounces any attempt at passive stretching and exercise $\left({ }^{2}\right)$. Many other forms of passive stretching are practised by pliysiotherapists. Patients with stiff elbows are encouraged to carry heavy weiglats or to hang from overheacl beams. while children are made to carry a heavy school case. Increasing stiffness is inevitable.

From this it will be gathered that passive stretching of the joint always defeats its own object. Instead of increasing the range of movement, it actually aggravates the stiffness. This is not the only sequel. It is often responsible for the formation of new bone around the joint, a condition known as myositis ossificans traumatica.
Traumatic myositis ossificans.-This complication results from tearing of the periosteum. It is encountered most often in children and adolescents because the periosteum of the young strips more easily. As a restilt of dislocation of the joint, muscle and capsule insertions into the periosteun must necessarily be torn; the condition is thus more common after dislocations than after fractures.

It must be emphasised that after reduction it is passive stretching and not active exercises which again displaces the periosteum. The ossification is therefore seen in front of the joint, in some cases because of avulsion of the brachialis muscle from the ulna, and the first sign of this complication is radiographic evidence of a shadow, which gradually becomes more dense as the mass becomes consolidated.

It is unnecessary in these cases to completely inımobilise the joint, but all passive movements must be avoided, and the patient slould be allowed to carry out active movements only. Recovery is fairly rapid, and absorption of the ossified material takes place.

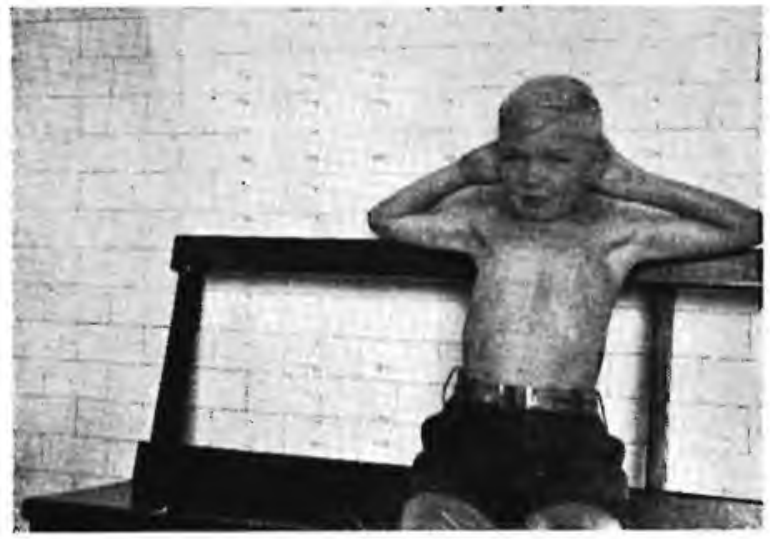

Fig. 3a.-3 months after injury-Full flexion recovered.

\section{SUMMARY.}

This short paper is published with the plea that all elbow-joint injuries should be approached with respect. The treatment, once the injury is referrel from the medical practitioner, is one of active movements and active movements only. Massage, passive stretching and manipulation should be avoided at all costs. In the very young, active movements can be cncouraged by the use of suitable toys. The acconpanying photographs and $X$-rays sloow that even in a badly injured and displaced joint, full range of flexion and extension can be obtained by active movements. The physiotherajist must exercise a great deal of batience, for it takes i4 days at least to accomplish 5 degrees of extension ( $\left.{ }^{3}\right)$. The temptation to hurry this on by manipulation and passive stretching must be constantly avoided. Thus a thorougl knowledge of the causes of a stiff elbow joint will help to avoid the tragedy of complete stiffness. which one comes across so often to-day.

\section{References :}

I. Watson-Jones "Adhesions of Joints and Injury," B.M.J.. May 1936, 925 .

2. Watson-Jones "Fractures and Joint Injuries," Vol. I. I944.

3. Walter Mercer, "Orthopaedic Surgery," I945. 